\title{
Mini-Review
}

Ahmad Adlie Shamsuri*, Khalina Abdan, and Siti Nurul Ain Md. Jamil

\section{Properties and applications of cellulose regenerated from cellulose/imidazolium-based ionic liquid/co-solvent solutions: A short review}

https://doi.org/10.1515/epoly-2021-0086

received September 25, 2021; accepted October 26, 2021

\begin{abstract}
An improvement of ecological conscience currently has increased the consciousness of researchers in reducing the processing time and cost of solvent for the dissolution of cellulose. Latterly, ionic liquids have been employed to process cellulose as they are recyclable and nonvolatile. Besides that, biopolymers such as chitosan, chitin, starch, protein, and cellulose acetate can also be processed by using ionic liquids for diverse applications. In this short review, examples of imidazolium-based ionic liquids that are commonly used for the dissolution of cellulose are implied. Furthermore, examples of organic liquids that are utilized as co-solvents for ionic liquids were revealed. In addition, examples of imidazolium-based ionic liquid/co-solvent mixtures utilized in the dissolution of cellulose and other biopolymers are also demonstrated. The properties and applications of cellulose and its blends regenerated from different types of cellulose/imidazolium-based ionic liquid/co-solvent solutions are also shortly reviewed. The information acquired from this review gives a better understanding of the changes in the properties of regenerated cellulose and regenerated cellulose blends. In addition, this short review serves as a model basis for the creation of novel applications of regenerated cellulose and regenerated cellulose blends by utilizing imidazolium-based ionic liquid/co-solvent mixtures.
\end{abstract}

\footnotetext{
* Corresponding author: Ahmad Adlie Shamsuri, Laboratory of Biocomposite Technology, Institute of Tropical Forestry and Forest Products, Universiti Putra Malaysia, UPM Serdang 43400, Selangor, Malaysia, e-mail: adlie@upm.edu.my

Khalina Abdan: Laboratory of Biocomposite Technology, Institute of Tropical Forestry and Forest Products, Universiti Putra Malaysia, UPM Serdang 43400, Selangor, Malaysia

Siti Nurul Ain Md. Jamil: Department of Chemistry, Faculty of Science, Universiti Putra Malaysia, UPM Serdang 43400, Selangor, Malaysia; Centre of Foundation Studies for Agricultural Science, Universiti Putra Malaysia, UPM Serdang 43400, Selangor, Malaysia
}

Keywords: cellulose, regeneration, imidazolium, ionic liquid, co-solvent

\section{Introduction}

Lately, the use of ionic liquids in the processing of biopolymers has been widespread due to the environmental awareness and development of the numerous ionic liquid industries. The application of ionic liquids for the processing of cellulose is a positive approach because they can be recycled. Ionic liquids are organic salts that usually have a melting point temperature lower than $100^{\circ} \mathrm{C}$. Ionic liquids also have a very low vapor pressure; therefore, they possess a nonvolatile character and are considered green solvents. Furthermore, ionic liquids have beneficial solvent properties, for example, nonflammability, high polarity, good electrical conductivity, and high thermal stability. But, the thermal stability of ionic liquids is mainly dependent on the anion (1). Ionic liquids can dissolve most organic materials, including biopolymers and some inorganic materials (2). They also have excellent solubility with many organic solvents. In addition, ionic liquids can be tailored as needed by some applications. Many types of ionic liquids have been successfully synthesized; the most common ionic liquids exploited in polymer science are imidazolium-based ionic liquids $(3,4)$. Table 1 shows examples of imidazolium-based ionic liquids used in the dissolution of cellulose. It can be seen that ionic liquids based on allyl-, butyl-, diallyl-, and ethyl-methylimidazolium cations with chloride, acetate, or diethylphosphate counter anions are frequently applied, and this suggests that the combination of the cations and anions is significantly effective for the dissolution of cellulose. Figure 1 shows the chemical structures of AmimCl, BmimOAc, BmimCl, AAeimOAc, EmimOAc, EmimCl, and EmimDEP.

Table 2 displays examples of organic liquids utilized as co-solvents for imidazolium-based ionic liquids. It can be observed that most organic liquids are highly polar 
Table 1: Examples of imidazolium-based ionic liquids used in the dissolution of cellulose

\begin{tabular}{lll}
\hline lonic liquid & Abbreviation & References \\
\hline 1-Allyl-3-methylimidazolium chloride & AmimCl & $(5)$ \\
1-Butyl-3-methylimidazolium acetate & BmimOAC & $(6)$ \\
1-Butyl-3-methylimidazolium chloride & $\mathrm{BmimCl}$ & $(7)$ \\
1,3-Diallyl-2-ethylimidazolium acetate & $\mathrm{AAeimOAC}$ & $(8)$ \\
1-Ethyl-3-methylimidazolium acetate & EmimOAc & $(9)$ \\
1-Ethyl-3-methylimidazolium chloride & EmimCl & $(10)$ \\
1-Ethyl-3-methylimidazolium diethylphosphate & EmimDEP & $(11)$ \\
\hline
\end{tabular}

(a)<smiles>C=CCn1cc[n+](C)c1</smiles>

(c)<smiles>CCCCn1cc[n+](C)c1</smiles>

(b)<smiles>CCCCn1cc[n+](C)c1</smiles>

(d)<smiles>C=CCn1cc[n+](CC=C)c1CC</smiles>

(e)<smiles>CCn1cc[n+](C)c1</smiles>

(f)<smiles>CCn1cc[n+](C)c1</smiles>

$\mathrm{Cl}^{-}$

(g)

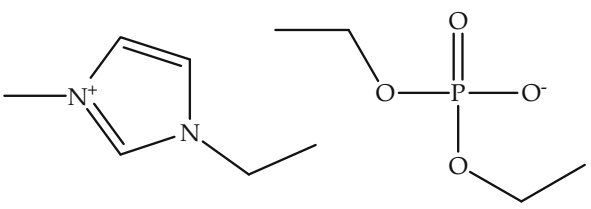

Figure 1: Chemical structures of ionic liquids: (a) AmimCl, (b) BmimOAc, (c) BmimCl, (d) AAeimOA, (e) EmimOAc, (f) EmimCl, and (g) EmimDEP.

Table 2: Examples of organic liquids utilized as co-solvents for imidazolium-based ionic liquids

\begin{tabular}{lll}
\hline Organic liquid & Abbreviation & References \\
\hline Dimethylacetamide & DMAC & $(12)$ \\
Dimethylformamide & DMF & $(13)$ \\
1,3-Dimethyl-2-imidazolidinone & DMI & $(14)$ \\
Dimethyl sulfoxide & DMSO & $(15)$ \\
Y-Butyrolactone & GBL & $(16)$ \\
Y-Valerolactone & GVL & $(17)$ \\
1-Methylimidazole & MIM & $(18)$ \\
\hline
\end{tabular}

solvents. Hence, they have good miscibility with ionic liquids that possess high polarity. Moreover, organic liquids are known as aprotic solvents, whereby they are neutral in charge, chemically inert, and typically have a low dielectric constant. In addition, the special feature of the organic liquids is that they own a high boiling point, and thus, the ionic liquid/co-solvent mixtures produced exhibit good thermal stability. Figure 2 displays the chemical structures of co-solvents DMAc, DMF, DMI, DMSO, GBL, GVL, and MIM. A previous study has shown that DMAc was utilized as a co-solvent for EmimOAc in the pretreatment of Salix gracilistyla Miq. (12). DMF was utilized as a co-solvent for EmimOAc in the preparation of cellulose fibers (13). DMI was utilized as a co-solvent for BmimOAc in the separation of polysaccharides (14). DMSO was utilized as a co-solvent for $\mathrm{BmimCl}$ in the preparation of carbon fiber precursors (15). GBL was utilized as a cosolvent for EmimOAc in the dissolution of cellulose (16). GVL was utilized as a co-solvent for $\mathrm{BmimCl}$ in the processing of polyaramids (17). Finally, MIM was utilized as a 


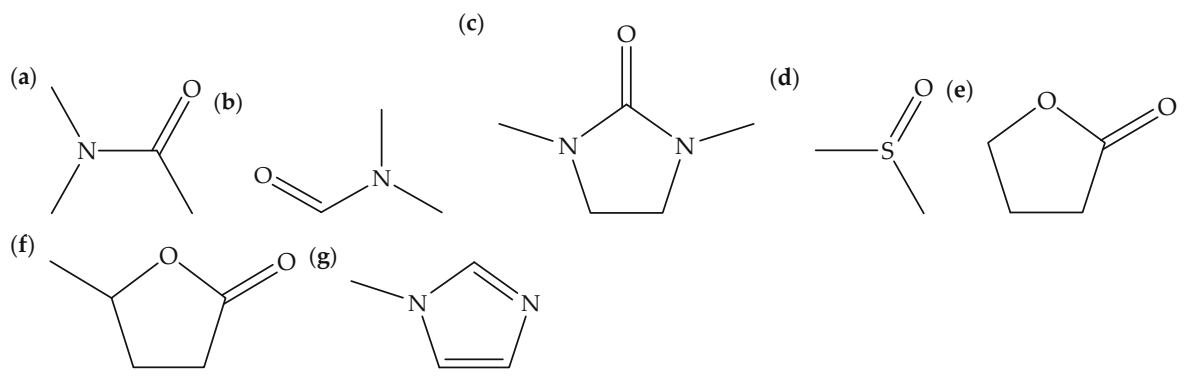

Figure 2: Chemical structures of co-solvents: (a) DMAc, (b) DMF, (c) DMI, (d) DMSO, (e) GBL, (f) GVL, and (g) MIM.

co-solvent for EmimOAc in the fractionation of lignin (18). From the previous studies, the utilization of co-solvents for imidazolium-based ionic liquids has become an important strategy for the development of well-designed natural and synthetic polymers.

In the last decade, several chemicals have been employed in the dissolution of cellulose with the purpose of diversifying the use of cellulose solution and regenerated cellulose. The utilization of ionic liquids as primary solvents can give a benefit since they can interact with cellulose without deteriorating the chemical structure of cellulose (19). Table 3 exhibits examples of imidazolium-based ionic liquid/co-solvent mixtures applied in the dissolution of cellulose and other biopolymers. It can be perceived that $\mathrm{AmimCl} / \mathrm{DMSO}, \mathrm{BmimOAc} / \mathrm{DMSO}, \mathrm{BmimCl} / \mathrm{DMSO}$, and EmimOAc/DMSO mixtures are regularly used as solvents for the

Table 3: Examples of imidazolium-based ionic liquid/co-solvent mixtures applied in the dissolution of cellulose and other biopolymers

\begin{tabular}{lll}
\hline $\begin{array}{l}\text { lonic liquid/co- } \\
\text { solvent mixture }\end{array}$ & Biopolymer & References \\
\hline AmimCl/DMSO & Cellulose & $(20-23)$ \\
BmimOAc/DMAc & Cellulose & $(24)$ \\
BmimOAc/DMF & Cellulose & $(24-26)$ \\
BmimOAc/DMI & Cellulose & $(25)$ \\
BmimOAc/DMSO & Cellulose, chitosan & $(24,25,27-29)$ \\
BmimOAc/GVL & Cellulose, chitin & $(30)$ \\
BmimCl/DMAc & Cellulose & $(21,31)$ \\
BmimCl/DMSO & Cellulose, starch, & $(15,21,22,32-34)$ \\
& protein, cellulose & \\
AAeimOAc/DMSO & acetate & $(8)$ \\
EmimOAc/DMAc & Cellulose & $(24,31,35)$ \\
EmimOAc/DMF & Cellulose & $(35-37)$ \\
EmimOAc/DMSO & Cellulose, chitosan & $(21,28,37-42)$ \\
EmimOAc/GBL & Cellulose & $(16)$ \\
EmimOAc/GVL & Cellulose & $(16)$ \\
EmimOAc/MIM & Cellulose & $(31,43)$ \\
EmimDEP/DMSO & Cellulose & $(11,44,45)$ \\
\hline
\end{tabular}

dissolution of cellulose. The addition of co-solvents can reduce the viscosity of ionic liquids and increase their volume. This leads to the decreased usage of ionic liquids and consequently decreases the cost of cellulose dissolution besides providing many choices of cellulose solutions. The ionic liquid/co-solvent mixtures can not only dissolve cellulose but also other biopolymers, such as chitosan, chitin, starch, zein protein, and cellulose acetate, and their chemical structures are shown in Figure 3. On top of that, as far as the authors are concerned, no short review has been done focusing on the properties and applications of regenerated cellulose and regenerated cellulose blends prepared from various sources of cellulose in imidazolium-based ionic liquid/co-solvent mixtures. This is the objective of doing a systematic review in this article. In addition, this short review is specific, and even though not inclusive, it is, however, relevant to other related studies.

\section{Isolation, dissolution, and regeneration of cellulose}

\subsection{Isolation of cellulose}

The utilization of biopolymers for packaging, semi-structural, and structural applications is a promising means for environment protection. Among biopolymers, cellulose is the most extensively studied biopolymer because it is easily available, renewable, biodegradable, and cheap. Currently, there are many kinds of bioresources that can be used as a source of cellulose. Table 4 presents examples of bioresources and imidazolium-based ionic liquid/co-solvent mixtures employed for the isolation of cellulose. It can be seen that bioresources such as bamboo pulp (8), cotton linters (20), cotton pulp (22), cotton stalk (23), Eucalyptus sawdust (37), flax fibers (38), Napier grass (46), oil palm frond (33), and pine wood bits (45) have been employed as cellulose sources. Cellulose was successfully isolated 
(a)

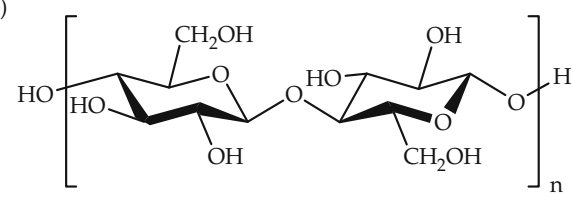

$(\mathbf{b}$

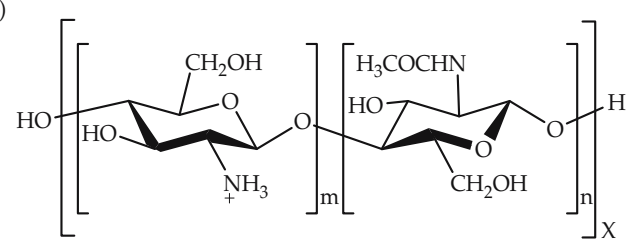

(c)

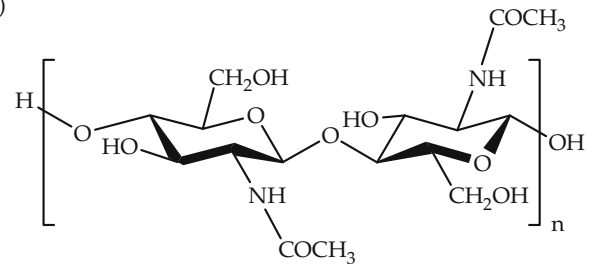

(d)

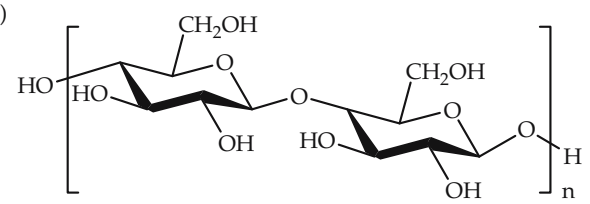

(e)
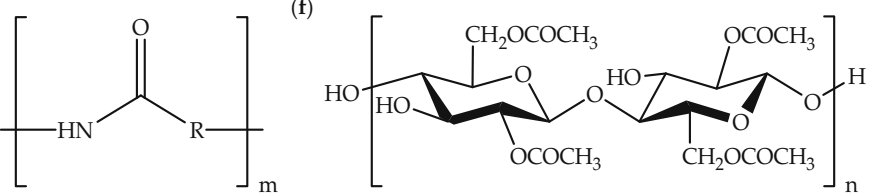

Figure 3: Chemical structures of biopolymers: (a) cellulose, (b) chitosan, (c) chitin, (d) starch, (e) zein protein, and (f) cellulose acetate.

Table 4: Examples of bioresources and imidazolium-based ionic liquid/co-solvent mixtures employed for the isolation of cellulose

\begin{tabular}{lll}
\hline Bioresource & $\begin{array}{l}\text { lonic liquid/co-solvent } \\
\text { mixture }\end{array}$ & References \\
\hline Bamboo pulp & AAeimOAC/DMSO & $(8)$ \\
Cotton linters & AmimCl/DMSO & $(20)$ \\
Cotton pulp & BmimCl/DMSO & $(22)$ \\
Cotton stalk & AmimCl/DMSO & $(23)$ \\
Eucalyptus sawdust & EmimOAc/DMF & $(37)$ \\
Flax fibers & EmimOAc/DMSO & $(38)$ \\
Napier grass & EmimOAc/DMSO & $(46)$ \\
Oil palm frond & BmimCl/DMSO & $(33)$ \\
Pine wood bits & EmimDEP/DMSO & $(45)$ \\
\hline
\end{tabular}

by employing different types of imidazolium-based ionic liquid/co-solvent mixtures through the dissolution process. Other approaches with aqueous ionic liquids have involved swelling and hydrolysis of natural fibers into cellulose nanocrystals (47-49). In Table 4, it can also be observed that imidazolium-based ionic liquids with chloride and acetate counter anions are commonly employed because they are capable of interacting with hydroxyl groups of cellulose via hydrogen bonding interaction in the dissolution mechanism (6). Moreover, co-solvent such as DMSO is frequently used compared to other co-solvents mentioned earlier because DMSO is less toxic and cheaper than other co-solvents. Therefore, the isolation of cellulose from bioresources on a large scale can be achieved by utilizing the imidazolium-based ionic liquid/DMSO mixtures without neglecting the toxicity and the cost of the solvents.

\subsection{Dissolution of cellulose}

Table 5 presents imidazolium-based ionic liquid/co-solvent mixtures, the ratio of mixtures, cellulose contents, temperatures, and times for the dissolution of cellulose. It can be noticed that the ratios of co-solvent to ionic liquid are equal to or less than that of ionic liquid, and this is due to the fact that the co-solvent ratios are ideal for mixtures. Interestingly, this can decrease the total cost of the solvents because a lesser proportion of ionic liquid is required without sacrificing the solubility of cellulose in the solvents. Moreover, the solubility does not change significantly in the cellulose conformation. Conversely, the dissolution of cellulose in imidazolium-based ionic liquid/co-solvent mixtures can be up to $23.6 \mathrm{wt} \%$ of cellulose content. This content is hard to achieve when using only ionic liquid as a result of its very high viscosity. With the presence of co-solvent, the viscosity of the ionic liquid decreases, thus allowing a higher solubility quantity of cellulose since co-solvent enhances the interaction between the ionic liquid and cellulose without participating in the interaction. Besides that, the dissolution of cellulose in the imidazolium-based ionic liquid/co-solvent mixtures is normally conducted at elevated temperatures, regardless of the type of the mixtures. Hence, the dissolution temperature plays an important role in the dissolution of cellulose. With an increase in temperature, the dissolution kinetics accelerate, consequently permitting a faster dissolution. In addition, the maximum dissolution temperature is $110^{\circ} \mathrm{C}$, which may prevent cellulose degradation. 
Table 5: Imidazolium-based ionic liquid/co-solvent mixtures, ratio of mixtures, cellulose contents, temperatures, and times for the dissolution of cellulose

\begin{tabular}{|c|c|c|c|c|c|}
\hline Ionic liquid/co-solvent mixture & Ratio of mixture & Cellulose content (wt\%) & Temperature $\left({ }^{\circ} \mathrm{C}\right)$ & Time (h) & References \\
\hline AmimCl/DMSO & $4: 1(w t)$ & 6 & 80 & 4 & $(22)$ \\
\hline BmimOAc/DMF & 1:1 (wt) & 8 & 40 & u & (26) \\
\hline BmimOAc/DMI & $1: 1(w t)$ & 10 & 110 & u & $(25)$ \\
\hline BmimOAc/DMSO & $1: 1(w t)$ & 10 & 110 & $\mathrm{u}$ & $(25)$ \\
\hline BmimOAc/GVL & $4: 1(\mathrm{~mol})$ & 8 & 90 & 3 & $(30)$ \\
\hline BmimCl/DMSO & $4: 1(w t)$ & 6 & 80 & 4 & $(22)$ \\
\hline AAeimOAc/DMSO & $3: 1(w t)$ & 12 & 110 & $\mathrm{u}$ & (8) \\
\hline EmimOAc/DMAc & $2: 1(w t)$ & 8.3 & $\mathrm{u}$ & $\mathrm{u}$ & (35) \\
\hline EmimOAc/DMF & $2: 1(w t)$ & 8.3 & u & u & (35) \\
\hline EmimOAc/DMSO & 1:1 (wt) & 15 & 80 & 72 & $(40)$ \\
\hline EmimOAc/GBL & 1:1 (vol) & 23 & 70 & $\mathrm{u}$ & (16) \\
\hline EmimOAc/GVL & 1:1 (vol) & 23 & 70 & $\mathrm{u}$ & $(16)$ \\
\hline EmimOAc/MIM & $5: 2(\mathrm{~mol})$ & 20 & 50 & 5 & (31) \\
\hline EmimDEP/DMSO & $7: 3(w t)$ & 23.6 & 100 & 6 & (11) \\
\hline
\end{tabular}

$\mathrm{wt}=$ weight, $\mathrm{mol}=$ molar, $\mathrm{vol}=$ volume, and $\mathrm{u}=$ unstated .

\subsection{Regeneration of cellulose}

Cellulose can be regenerated from cellulose/ionic liquid/co-solvent solutions by immersing them in a coagulation agent. This agent is anti-solvent because of its ability to reduce the solvent quality of ionic liquids. In contrast to co-solvent, the coagulation agent induces cellulose regeneration by removing ionic liquid since it is completely miscible with the coagulation agent at any ratio. During the coagulation process, the anions and cations of the ionic liquid rapidly move away from the cellulose solution to the coagulation agent; this causes the regeneration of the cellulose gel. After the elimination of the coagulation agent, the formation of the solid cellulose occurs. Table 6 presents examples of imidazoliumbased ionic liquid/co-solvent mixtures and coagulation agents used for the regeneration of cellulose. It can be observed that ethanol and water are regularly used as coagulation agents for cellulose regeneration. Generally, water is preferred as a coagulation agent because it is of low cost (6). Nonetheless, coagulation in water is clearly distinct from coagulation in alcohols with respect to the microstructures formed and the mechanisms that generate the microstructures (39). Conversely, the ionic liquid/cosolvent mixture can be recycled by evaporating a major quantity of coagulation agent under reduced pressure, followed by freeze-drying under vacuum pressure. In Table 6, it can also be seen that the coagulation agent such as carbon dioxide can be used to regenerate cellulose and remove ionic liquid/co-solvent mixtures (25). Long-chain cellulose with larger molecular weight is more easily to be surrounded by carbon dioxide molecules, leading to a poorer interaction with ionic liquid and thus resulting in an easier regeneration (50).

\section{Properties of cellulose and its blends regenerated from cellulose/imidazolium-based ionic liquid/co-solvent solutions}

\subsection{Properties of regenerated cellulose}

Table 7 presents the crystalline, chemical, and thermal properties of cellulose regenerated from cellulose/imidazolium-based ionic liquid/co-solvent solutions. The regenerated cellulose has been prepared from bamboo pulp/AAeimOAc/DMSO solution (8).

Table 6: Examples of imidazolium-based ionic liquid/co-solvent mixtures and coagulation agents used for the regeneration of cellulose

\begin{tabular}{lll}
\hline $\begin{array}{l}\text { Ionic liquid/co-solvent } \\
\text { mixture }\end{array}$ & Coagulation agent & References \\
\hline BmimOAC/DMF & Ethanol & $(26)$ \\
BmimOAC/DMI & Carbon dioxide & $(25)$ \\
BmimOAC/GVL & Ethanol & $(30)$ \\
AAeimOAC/DMSO & Water & $(8)$ \\
EmimOAC/DMAc & Ethanol & $(35)$ \\
EmimOAC/MIM & Water & $(31)$ \\
EmimDEP/DMSO & Water & $(11)$ \\
\hline
\end{tabular}


Table 7: Crystalline, chemical, and thermal properties of cellulose regenerated from cellulose/imidazolium-based ionic liquid/co-solvent solutions

\begin{tabular}{llllll}
\hline Cellulose source & Ionic liquid/co-solvent mixture & & Properties & \\
\cline { 3 - 5 } & & Crystalline & Chemical & Thermal & References \\
\hline Bamboo pulp & AAeimOAC/DMSO & $\nabla$ & $\Delta$ & $\nabla$ & $(8)$ \\
Cellulose powder & EmimOAc/DMF & $\nabla$ & $\nabla$ & $\nabla$ & $(35)$ \\
Cotton stalk & AmimCl/DMSO & $\nabla$ & $\nabla$ & $\Delta$ & $(23)$ \\
Microcrystalline cellulose & EmimOAc/DMSO & $\nabla$ & $\nabla$ & $\nabla$ & $(41)$ \\
Treated pine cellulose & EmimDEP/DMSO & $\nabla$ & $\nabla$ & $\nabla$ & $(45)$ \\
\hline
\end{tabular}

The symbol " $\nabla$ " corresponds to a decrease in the properties, and " $\Delta$ " corresponds to an increase in the properties.

The crystalline, chemical, and thermal properties of the regenerated cellulose were characterized by using an X-ray diffractometer, Fourier transform infrared spectrometer, and thermal gravimetric analyzer. The crystalline property, such as the intensity of the X-ray diffraction peaks of the regenerated cellulose, is weaker compared to that of the original bamboo pulp cellulose. This indicated that the crystallinity of cellulose decreased because its crystalline structure changed from I to II. Furthermore, the chemical property, such as the infrared absorption band of the $\mathrm{O}-\mathrm{H}$ stretching vibration, of the regenerated cellulose shifted to a higher wavenumber region compared to the $\mathrm{O}-\mathrm{H}$ stretching vibration of the original bamboo pulp cellulose because the breaking of hydrogen bonding weakened the bonding force during the dissolution of cellulose. Conversely, the thermal property, such as the decomposition temperature of the regenerated cellulose, is lower than that of the original bamboo pulp cellulose. This was ascribed to the decreased number of hydrogen bonding between cellulose chains and crystallinity, which decreased the thermal stability of the regenerated cellulose (8). Hence, it can be deduced that the utilization of the AAeimOAc/DMSO mixture gives regenerated cellulose with a low crystallinity and low thermal decomposition behavior.

Meanwhile, the regenerated cellulose fibers have been prepared from cellulose powder/EmimOAc/DMF solutions (35). The crystalline, chemical, and thermal properties of the regenerated cellulose fibers were characterized by using an X-ray diffractometer, Fourier transform infrared spectrometer, and thermal gravimetric analyzer. The crystalline property, such as the degree of crystallinity of the regenerated cellulose fibers, is significantly lesser compared to that of the cellulose powder because the rapid coagulation of cellulose from the ionic liquid did not permit sufficient time to create hydrogen bonding. Besides, the chemical property such as the infrared absorption bands for EmimOAc and DMF is not detected in the regenerated cellulose fibers. This exhibited that the residue of the ionic liquid and the co-solvent in the fibers was fully removed by the coagulation agent. On top of that, the thermal property such as the decomposition temperature of the regenerated cellulose fibers is slightly smaller than that of the cellulose powder. This was caused by the influence of the cellulose crystallinity on the thermal stability of the microstructure of the regenerated cellulose fibers and consecutively resulted in the lower decomposition enthalpy (35). Therefore, it can be concluded that the utilization of the EmimOAc/DMF mixture grants regenerated cellulose fibers with a less degree of crystallinity and slightly low thermal stability.

The regenerated cellulose aerogels have been prepared from cotton stalk/AmimCl/DMSO solutions (23). The crystalline, chemical, and thermal properties of the regenerated cellulose aerogels were characterized by using an X-ray diffractometer, Fourier transform infrared spectrometer, and thermal gravimetric analyzer. The crystalline property such as the crystallinity index of the regenerated cellulose aerogels is lower than that of the raw cotton stalk. This was caused by the reorientation of cellulose chains in the form of parallel chains. Moreover, the chemical property, such as the infrared absorption bands of the $\mathrm{O}-\mathrm{H}$ stretching vibrations, of the regenerated cellulose aerogels shifted to lower wavenumber regions compared to the $\mathrm{O}-\mathrm{H}$ stretching vibration of the raw cotton stalk. This was attributed to the increase of hydrogen bonding between cellulose and other biopolymer components, resulting in the restriction of these biopolymers in the cellulose matrix. Besides that, the thermal property such as the decomposition temperature of the regenerated cellulose aerogels increased by up to $1.8 \%$ in comparison to the raw cotton stalk. This was due to the porous structure of the aerogels, which increased the thermal stability of the regenerated cellulose aerogels (23). Thus, it can be inferred that the utilization of the AmimCl/DMSO mixture provides regenerated cellulose aerogels with a low crystallinity index and high thermal decomposition behavior. 
Besides that, the regenerated cellulose hollow fibers have been prepared from microcrystalline cellulose/EmimOAc/DMSO solutions (41). The crystalline, chemical, and thermal properties of the regenerated cellulose hollow fibers were characterized by using an X-ray diffractometer, Fourier transform infrared spectrometer, and thermal gravimetric analyzer. The crystalline property such as the degree of crystallinity of the regenerated cellulose hollow fibers is lower compared to that of the raw microcrystalline cellulose. This was because the initial crystalline structure was partly changed during the dissolving through interaction with ionic liquid. Moreover, the chemical property such as the infrared absorption bands of the $\mathrm{C}-\mathrm{O}-\mathrm{C}$ and $\mathrm{C}-\mathrm{O}$ stretching vibrations, of the regenerated cellulose hollow fibers shifted to lower wavenumber regions in comparison to the $\mathrm{C}-\mathrm{O}-\mathrm{C}$ and $\mathrm{C}-\mathrm{O}$ stretching vibrations of the raw microcrystalline cellulose. This was due to the cellulose I crystalline structure in the raw microcrystalline cellulose was transformed to cellulose II in the regenerated cellulose hollow fibers. In addition, the thermal property such as the decomposition temperature of the regenerated cellulose hollow fibers is lesser than that of the raw microcrystalline cellulose. This was because of the decreased crystallinity of cellulose II and the transition of the crystalline structure (41). So, it can be inferred that the utilization of the EmimOAc/DMSO mixture provides regenerated cellulose hollow fibers with a low degree of crystallinity and less thermal decomposition behavior.

Conversely, the regenerated cellulose film has been prepared from treated pine cellulose/EmimDEP/DMSO solution (45). The crystalline, chemical, and thermal properties of the regenerated cellulose film were characterized by using an X-ray diffractometer, Fourier transform infrared spectrometer, and thermal gravimetric analyzer. The crystalline property such as the X-ray diffraction peaks of the regenerated cellulose film exhibited the presence of broad crystalline peaks compared to that of the treated pine cellulose. This was because the cellulose was changed from cellulose I to cellulose II during the dissolution and regeneration. Furthermore, the chemical property, such as the infrared absorption band of the $\mathrm{O}-\mathrm{H}$ stretching vibration, of the regenerated cellulose film shifted to a lower wavenumber region in comparison to the $\mathrm{O}-\mathrm{H}$ stretching vibration of the treated pine cellulose. This was because the connection between the hydroxyl groups was decreased, and subsequently, the force of the intermolecular hydrogen bonding was reduced. In addition, the thermal property such as the decomposition temperature of the regenerated cellulose film is lower than that of the treated pine cellulose. This was attributed to the decrease in crystallinity of the regenerated cellulose film, which lowered its thermal stability (45). Consequently, it can be deduced that the utilization of the EmimDEP/DMSO mixture gives broad crystalline peaks and low thermal stability to the regenerated cellulose film.

\subsection{Properties of regenerated cellulose blends}

Table 8 displays the crystalline, chemical, and thermal properties of cellulose blends regenerated from cellulose blend/imidazolium-based ionic liquid/co-solvent solutions. The regenerated cellulose/agar blend aerogels have been prepared from microcrystalline cellulose/agar/ BmimCl/DMSO solutions (34). The chemical and thermal property of the regenerated cellulose/agar blend aerogels were characterized by using Fourier transform infrared spectrometer and differential scanning calorimeter. The chemical property, such as the infrared absorption band of the $\mathrm{O}-\mathrm{H}$ stretching vibration, of the regenerated cellulose/agar blend aerogel shifted to a higher wavenumber region in comparison

Table 8: Crystalline, chemical, and thermal properties of cellulose blends regenerated from cellulose blend/imidazolium-based ionic liquid/co-solvent solutions

\begin{tabular}{|c|c|c|c|c|c|}
\hline \multirow[t]{2}{*}{ Cellulose blend } & \multirow[t]{2}{*}{ Ionic liquid/co-solvent mixture } & \multicolumn{3}{|c|}{ Properties } & \multirow[t]{2}{*}{ References } \\
\hline & & Crystalline & Chemical & Thermal & \\
\hline Cellulose/agar & BmimCl/DMSO & 0 & $\Delta$ & $\nabla$ & (34) \\
\hline Cellulose/chitin & $\mathrm{BmimOAc} / \mathrm{GVL}$ & $\nabla$ & $\nabla$ & 0 & (30) \\
\hline Cellulose/polyamide & EmimOAc/DMSO & 0 & $\Delta$ & $\nabla$ & $(42)$ \\
\hline Cellulose/polylactic acid & $\mathrm{BmimOAc/DMF}$ & $\nabla$ & $\Delta$ & $\Delta$ & $(26)$ \\
\hline Cotton/polyacrylonitrile & BmimCl/DMSO & 0 & $\Delta$ & $\nabla$ & $(15)$ \\
\hline
\end{tabular}

The symbol " $\nabla$ " corresponds to a decrease in the properties, and " $\Delta$ " corresponds to an increase in the properties, while " 0 " describes not available. 
to the $\mathrm{O}-\mathrm{H}$ stretching vibration of the raw microcrystalline cellulose. This was due to the formation of intermolecular hydrogen bonding between the hydroxyl groups of cellulose and the hydroxyl groups of agar. This indicated the presence of intermolecular interaction between cellulose and agar, which increased the miscibility between them. Nonetheless, the thermal property such as the melting point temperature of the regenerated cellulose/agar blend aerogel is considerably lower compared to that of the pristine agar. This was ascribed to the thermodynamic factor such as polymer-polymer interaction, which exhibited the depression in the melting temperature (34). Thus, it can be inferred that the utilization of the BmimCl/DMSO mixture provides regenerated cellulose/agar blend aerogels with a good interaction of their components and low melting temperature.

Meanwhile, the regenerated cellulose/chitin blend films have been prepared from microcrystalline cellulose/chitin/BmimOAc/GVL solutions (30). The crystalline and chemical properties of the regenerated cellulose/chitin blend films were characterized by using an X-ray diffractometer and Fourier transform infrared spectrometer. The crystalline property such as the X-ray diffraction peaks of the regenerated cellulose/chitin blend films showed only flat and broad diffraction peaks compared to those of the raw microcrystalline cellulose and chitin. This was attributed to the formation of cellulose II in the amorphous state as well as the transition from the $\alpha$-chitin to the $\beta$-chitin polymorph after regeneration. Moreover, the crystallinity index and crystallite size of the regenerated cellulose/chitin blend films are lower than those of the raw microcrystalline cellulose and chitin. This was due to the dissolution process, whereby crystalline structures of cellulose and chitin were destroyed. Besides that, the chemical property such as the intensity of the infrared absorption bands of the regenerated cellulose/chitin blend films is lesser in comparison to those of the raw microcrystalline cellulose and chitin. This also showed the change of the crystalline structures after regeneration (30). Hence, it can be deduced that the utilization of the BmimOAc/GVL mixture gives regenerated cellulose/chitin blend films with a low crystallinity index and crystallite size.

The regenerated cellulose/polyamide blend films have been prepared from microcrystalline cellulose/polyamide6/EmimOAc/DMSO solutions (42). The chemical and thermal properties of the regenerated cellulose/polyamide blend films were characterized by using Fourier transform infrared spectrometer and differential scanning calorimeter. The chemical property, such as the infrared absorption bands of the $\mathrm{C}-\mathrm{O}$ stretching vibrations, of the regenerated cellulose/polyamide blend films shifted to higher wavenumber regions compared to the $\mathrm{C}-\mathrm{O}$ stretching vibration of the raw microcrystalline cellulose. Furthermore, the infrared absorption bands of the $\mathrm{N}-\mathrm{H}$ deformation vibrations of the regenerated cellulose/polyamide blend films shifted to higher wavenumber regions in comparison to the $\mathrm{N}-\mathrm{H}$ deformation vibration of the pure polyamide- 6 . This was because of the interactions of the $\mathrm{N}-\mathrm{H}$ group with the cellulose backbone. Nevertheless, the thermal property such as the melting point temperature of the regenerated cellulose/polyamide blend films is substantially lower than that of the pure polyamide- 6 . This was owing to the intermolecular interactions between the cellulose and polyamide chains $(42,51)$. So, it can be inferred that the utilization of the EmimOAc/DMSO mixture provides regenerated cellulose/polyamide blend films with good interactions of their components and low melting temperature.

Besides that, the regenerated cellulose/polylactic acid blend films have been prepared from microcrystalline cellulose/polylactic acid/BmimOAc/DMF solutions (26). The crystalline, chemical, and thermal properties of the regenerated cellulose/polylactic acid blend films were characterized by using an X-ray diffractometer, Fourier transform infrared spectrometer, and thermal gravimetric analyzer. The crystalline property, such as the X-ray diffraction peaks of the regenerated cellulose/polylactic acid blend films, disappeared than those of the raw microcrystalline cellulose and polylactic acid film. This was attributed to the amorphous state present in the blend films. On top of that, the chemical property, such as the infrared absorption bands of the $\mathrm{O}-\mathrm{H}$ stretching vibrations, of the regenerated cellulose/polylactic acid blend films shifted to higher wavenumber regions in comparison to the $\mathrm{O}-\mathrm{H}$ stretching vibration of the pure cellulose film. This was due to the presence of a hydrogen bonding interaction between the hydroxyl groups of cellulose and the carbonyl groups of polylactic acid, which decreased the hydrogen bonding interaction between the cellulose chains. In addition, the thermal property such as the decomposition temperature of the regenerated cellulose/polylactic acid blend films increased by up to $33 \%$ compared to the pure cellulose film. This was ascribed to the presence of interaction between them (52), which induced the good thermal stability of the blend films (26). Consequently, it can be deduced that the utilization of the BmimOAc/DMF mixture gives regenerated cellulose/polylactic acid blend films with a good interaction of their components and high thermal decomposition behavior.

Conversely, the regenerated cellulose/polyacrylonitrile blend fibers have been prepared from raw cotton/polyacrylonitrile/BmimCl/DMSO solutions (15). The chemical and thermal properties of the regenerated 
cellulose/polyacrylonitrile blend fibers were characterized by using Fourier transform infrared spectrometer and thermal gravimetric analyzer. The chemical property, such as the intensity of the infrared absorption bands of the hydroxyl stretching vibrations, of the regenerated cellulose/polyacrylonitrile blend fibers is higher in comparison to the hydroxyl stretching vibration of the pure polyacrylonitrile. This was caused by the addition of the natural biopolymer, which has a substantial band in this wavenumber region. However, the intensity of the infrared absorption bands of the amide stretching vibrations, of the regenerated cellulose/polyacrylonitrile blend fibers, is lower compared to the amide stretching vibration of the pure polyacrylonitrile. Conversely, the thermal property such as the decomposition temperature of the regenerated cellulose/polyacrylonitrile blend fibers is significantly lower than that of the pure polyacrylonitrile. This phenomenon is similar to lignin/polyacrylonitrile blends that showed a decrease in the decomposition temperature (15). Therefore, it can be concluded that the utilization of the BmimCl/DMSO mixture grants regenerated cellulose/polyacrylonitrile blend fibers with a high hydroxyl stretching vibration intensity and low thermal decomposition behavior.

\section{Applications of cellulose and its blends regenerated from cellulose/imidazolium-based ionic liquid/co-solvent solutions}

\subsection{Applications of regenerated cellulose}

Table 9 presents examples of applications of cellulose regenerated from cellulose/imidazolium-based ionic liquid/co-solvent solutions. It can be seen that the imidazolium-based ionic liquid/DMSO mixtures are often utilized in the development of regenerated cellulose applications because DMSO is less toxic and cheaper than other cosolvents, as previously mentioned. On top of that, the regenerated cellulose was applied in the manufacturing of continuous and sustainable filaments (44), and the mechanical properties of the filaments are higher than other commercial regenerated cellulose fibers. Moreover, the regenerated cellulose was applied in the preparation of nanofibers (35) that have finer fiber diameters, better web uniformity, higher crystallinity, and better thermal stability. In addition, the regenerated cellulose was applied in the fabrication of aerogels (23), and this approach is not only environmentally friendly but also advantageous in creating a more commercially viable ionic liquid-based system. Besides that, the regenerated cellulose was also applied in the preparation of hollow fibers (41), and these fibers can be used as a precursor to develop hollow fiber carbon membranes for gas separation. Furthermore, the regenerated cellulose was applied in the production of high-performance fibers (11) by using high concentrations of cellulose with low molecular weight, and the processing time and cost are also significantly reduced. In addition, the regenerated cellulose was applied in the production of 3D-printed structures (29); therefore, this renewable biopolymer can replace the synthetic nonbiodegradable polymers in the 3D additive manufacturing process for producing single and multiple components.

\subsection{Applications of regenerated cellulose blends}

Table 10 presents examples of applications of cellulose blends regenerated from cellulose blend/imidazoliumbased ionic liquid/co-solvent solutions. It can be observed that cellulose can be blended with natural biopolymers and synthetic polymers in imidazolium-based ionic liquid/cosolvent mixtures for a wide variety of applications. Conversely, the regenerated cellulose/agar blends were applied in the fabrication of blend aerogels (34), and the bulk density

Table 9: Examples of applications of cellulose regenerated from cellulose/imidazolium-based ionic liquid/co-solvent solutions

\begin{tabular}{llll}
\hline Cellulose & Ionic liquid/co-solvent mixture & Application & References \\
\hline Cellulose nanofibers & EmimDEP/DMSO & Continuous filaments & $(44)$ \\
Cellulose powder & EmimOAc/DMF & Nanofibers & $(35)$ \\
Cotton stalk & AmimCl/DMSO & Aerogels & $(23)$ \\
Microcrystalline cellulose & EmimOAC/DMSO & Hollow fibers & $(41)$ \\
Microcrystalline cellulose & EmimDEP/DMSO & High-performance fibers & $(11)$ \\
Microgranular cellulose & BmimOAc/DMSO & 3D-printed structures & $(29)$ \\
\hline
\end{tabular}


Table 10: Examples of applications of cellulose blends regenerated from cellulose blend/imidazolium-based ionic liquid/co-solvent solutions

\begin{tabular}{llll}
\hline Cellulose blend & lonic liquid/co-solvent mixture & Application & References \\
\hline Cellulose/agar & BmimCl/DMSO & Blend aerogels & $(34)$ \\
Cellulose/chitin & BmimOAc/GVL & Biodegradable films & $(30)$ \\
Cellulose/chitosan & EmimOAc/DMSO & Porous macrospheres & $(28)$ \\
Cellulose/polyacrylonitrile & BmimCl/DMSO & Blend fibers & $(15)$ \\
Cellulose/polyamide & EmimOAc/DMSO & Blend films & $(42)$ \\
Cellulose/polylactic acid & BmimOAc/DMF & Biocompatible films & $(26)$ \\
\hline
\end{tabular}

of the aerogels is lower compared to their individual components because of the small pore size diameters. Furthermore, the regenerated cellulose/chitin blends were applied in the preparation of biodegradable films (30) that can be used in packaging, medical, or textile uses substituting fossil resource-based substances. In addition, the regenerated cellulose/chitosan blends were applied in the production of porous macrospheres (28), and these macrospheres are able to remove significant amounts of copper ions contaminants from water. Besides that, the regenerated cellulose/polyacrylonitrile blends were also applied in the creation of blend fibers (15) that can be employed as low-cost environmentally friendly precursors for carbon fiber. Moreover, the regenerated cellulose/polyamide blends were applied in the preparation of blend films (42), and these films have the potential to be used in textiles or technical usages owing to their homogeneous morphology. In addition, the regenerated cellulose/polylactic acid blends were applied in the fabrication of biocompatible films (26) that have possible functions in the biomedical field. The films are appropriate for large-scale production due to their very simple preparation procedure and quite mild manufacturing condition.

\section{Conclusion}

Examples of imidazolium-based ionic liquid/co-solvent mixtures, coagulation agents, as well as isolation, dissolution, and regeneration processes of cellulose were shortly reviewed in this article. The significant properties, for example, the crystalline, chemical, and thermal properties, of the regenerated cellulose and regenerated cellulose blends were also described in this short review. The employment of co-solvents has reduced the viscosity of ionic liquids and decreased the total cost of cellulose solutions without sacrificing the cellulose solubility and conformation. In addition, DMSO is commonly employed as a co-solvent for imidazolium-based ionic liquids as it is less toxic and cheaper than other co-solvents. Conversely, the utilization of imidazolium-based ionic liquid/co-solvent mixtures can effectively decrease the crystalline and thermal properties of the regenerated cellulose and regenerated cellulose blends. However, the utilization of imidazolium-based ionic liquid/co-solvent mixtures can efficiently increase the chemical property of the regenerated cellulose blends by creating intermolecular interactions between the cellulose and other polymer chains. The regenerated cellulose can be applied in the preparation of fibers, aerogels, etc. Meanwhile, the regenerated cellulose blends can be applied in the fabrication of blend aerogels, blend films, and others. This short review may be useful for the dissolution and regeneration of cellulose and its blends in diversifying their applications by utilizing imidazolium-based ionic liquid/co-solvent mixtures.

Acknowledgments: The authors would like to thank Dr. Krzysztof Debniak the Managing Editor of e-Polymers for assisting the authors to publish this short review.

Funding information: This short review was supported by the Universiti Putra Malaysia under the Grant Putra IPM Scheme (project number: GP-IPM/2021/9697900).

Author contributions: Ahmad Adlie Shamsuri: writing original draft, formal analysis, conceptualization; Siti Nurul Ain Md. Jamil: writing - review and editing, validation, project administration; Khalina Abdan: funding acquisition, resources, supervision.

Conflict of interest: The authors state no conflict of interest.

\section{References}

(1) Xue Z, Qin L, Jiang J, Mu T, Gao G. Thermal, electrochemical and radiolytic stabilities of ionic liquids. Phys Chem Chem Phys. 2018;20:8382-402. 
(2) Shamsuri AA, Md. Jamil SNA. Compatibilization effect of ionic liquid-based surfactants on physicochemical properties of PBS/Rice starch blends: an initial study. Materials (Basel). 2020;13:1885

(3) Abushammala $\mathrm{H}$, Mao J. A review on the partial and complete dissolution and fractionation of wood and lignocelluloses using imidazolium ionic liquids. Polym (Basel). 2020;12:195.

(4) Shamsuri AA, Daik R, Md. Jamil SNA. A succinct review on the PVDF/imidazolium-based ionic liquid blends and composites: preparations, properties, and applications. Processes. 2021;9:761.

(5) Pang J, Liu X, Zhang X, Wu Y, Sun R. Fabrication of cellulose film with enhanced mechanical properties in ionic liquid 1-Allyl-3-methylimidaxolium chloride (Amim Cl). Mater (Basel). 2013;6:1270-84.

(6) Shamsuri AA, Abdan K, Kaneko T. A concise review on the physicochemical properties of biopolymer blends prepared in ionic liquids. Molecules. 2021;26:216.

(7) Tanimura K, Ooe Y, Suga K. Effective concentration of ionic liquids for enhanced saccharification of cellulose. Chem Eng. 2018;2:47.

(8) Zhang H, Xu Y, Li Y, Lu Z, Cao S, Fan M, et al. Facile cellulose dissolution and characterization in the newly synthesized 1,3diallyl-2-ethylimidazolium acetate ionic liquid. Polym (Basel). 2017;9:1-12.

(9) Ślusarczyk C, Fryczkowska B. Structure - property relationships of pure cellulose and GO/CEL membranes regenerated from ionic liquid solutions. Polym (Basel). 2019;11:1178.

(10) Spiridon I, Andrei IM, Anghel N, Dinu MV, Ciubotaru BI. Development and characterization of novel cellulose composites obtained in 1-ethyl-3-methylimidazolium chloride used as drug delivery systems. Polym (Basel). 2021;13:2176.

(11) Zhu C, Koutsomitopoulou AF, Eichhorn SJ, van Duijneveldt JS, Richardson RM, Nigmatullin R, et al. High Stiffness cellulose fibers from low molecular weight microcrystalline cellulose solutions using DMSO as co-solvent with ionic liquid. Macromol Mater Eng. 2018;303:29-34.

(12) Han S, Park C, Febrianto F, Kim N, Lee S. Pretreatment with [EMIM]AC/DMAC co-solvent to improve enzymatic saccharification of pussy willow (Salix gracilistyla Miq.). Bioresources. 2020;15:187-98.

(13) Hong JH, Ku MK, Ahn Y, Kim HJ, Kim H. Air-gap spinning of cellulose/ionic liquid solution and its characterization. Fibers Polym. 2013;14:2015-9.

(14) Hou Q, Li W, Ju M, Liu L, Chen Y, Yang Q, et al. Separation of polysaccharides from rice husk and wheat bran using solvent system consisting of BMIMOAC and DMI. Carbohydr Polym. 2015;133:517-23.

(15) Byrne N, Leblais A, Fox B. Preparation of polyacrylonitrilenatural polymer composite precursors for carbon fiber using ionic liquid co solvent solutions. J Mater Chem A. 2014;2:3424-9.

(16) Gale E, Wirawan RH, Silveira RL, Pereira CS, Johns MA, Skaf MS, et al. Directed discovery of greener cosolvents: new cosolvents for use in ionic liquid based organic electrolyte solutions for cellulose dissolution. ACS Sustain Chem Eng. 2016;4:6200-7.

(17) Winters J, Winters J. $\gamma$-Valerolactone-based organic electrolyte solutions: a benign approach to polyaramid dissolution and processing. Green Chem. 2020;22:6127-36.
(18) Bylin S, Wells T, Sun Q, Ragauskas A, Theliander H. Lignin structure and aggregation behavior in a two- component ionic liquid solvent system. BioResour. 2014;9:6002-18.

(19) Shamsuri AA, Md. Jamil SNA, Abdan K. A brief review on the influence of ionic liquids on the mechanical, thermal, and chemical properties of biodegradable polymer composites. Polym (Basel). 2021;13:2597.

(20) Bioni TA, Arêas EPG, Couto LG, Favarin G, El Seoud OA. Dissolution of cellulose in mixtures of ionic liquid and molecular solvents: relevance of solvent-solvent and cellulosesolvent interactions. Nord Pulp Pap Res J. 2015;30:105-11.

(21) Gericke M, Liebert T, Seoud OAE, Heinze T. Tailored media for homogeneous cellulose chemistry: Ionic liquid/co-solvent mixtures. Macromol Mater Eng. 2011;296:483-93.

(22) Lv Y, Wu J, Zhang J, Niu Y, Liu CY, He J, et al. Rheological properties of cellulose/ionic liquid/dimethylsulfoxide (DMSO) solutions. Polym (Guildf). 2012;53:2524-31.

(23) Mussana H, Yang X, Tessima M, Han F, Iqbal N, Liu L. Preparation of lignocellulose aerogels from cotton stalks in the ionic liquidbased co-solvent system. Ind Crop Prod. 2018;113:225-33.

(24) Zhang L, Huang C, Zhang C, Pan H. Swelling and dissolution of cellulose in binary systems of three ionic liquids and three cosolvents. Cellulose. 2021;28:4643-53.

(25) Sun X, Chi Y, Mu T. Studies on staged precipitation of cellulose from an ionic liquid by compressed carbon dioxide. Green Chem. 2014;16:2736-44.

(26) Xu A, Wang Y, Gao J, Wang J. Facile fabrication of a homogeneous cellulose/polylactic acid composite fi Im with improved biocompatibility, biodegradability and mechanical. Green Chem. 2019;21:4449-56.

(27) Andanson JM, Bordes E, Devémy J, Leroux F, Pádua AAH, Gomes MFC. Understanding the role of co-solvents in the dissolution of cellulose in ionic liquids. Green Chem. 2014;16:2528-38.

(28) Wittmar ASM, Böhler H, Kayali AL, Ulbricht M. One-step preparation of porous cellulose/chitosan macro-spheres from ionic liquid-based solutions. Cellulose. 2020;27:5689-705.

(29) Gunasekera DH, Kuek S, Hasanaj D, He Y, Tuck C, Croft AK, et al. Three dimensional ink-jet printing of biomaterials using ionic liquids and co-solvents. Faraday Discuss. 2019;190:509-23.

(30) Duan Y, Freyburger A, Kunz W, Zollfrank C. Cellulose and chitin composite materials from an ionic liquid and a green co-solvent. Carbohydr Polym. 2018;192:159-65.

(31) Olsson C, Hedlund A, Idström A, Westman G. Effect of methylimidazole on cellulose/ionic liquid solutions and regenerated material therefrom. J Mater Sci. 2014;49:3423-33.

(32) Deng S, Cheng J, Guo X, Jiang L, Zhang J. Fiber spinning of polyacrylonitrile grafted soy protein in an ionic liquid/DMSO mixture solvent. J Polym Env. 2014;22:17-26.

(33) Mahmood H, Moniruzzaman M, Iqbal T, Yusup S. Effect of ionic liquids pretreatment on thermal degradation kinetics of agroindustrial waste reinforced thermoplastic starch composites. J Mol Liq. 2017;247:164-70.

(34) Shamsuri AA, Abdullah DK, Daik R. Fabrication of agar/biopolymer blend aerogels in ionic liquid and co-solvent mixture. Cellul Chem Technol. 2012;46:45-52.

(35) Ahn Y, Hu DH, Hong JH, Lee SH, Kim HJ, Kim H. Effect of cosolvent on the spinnability and properties of electrospun cellulose nanofiber. Carbohydr Polym. 2012;89:340-5. 
(36) Rein DM, Khalfin R, Szekely N, Cohen Y. True molecular solutions of natural cellulose in the binary ionic liquid-containing solvent mixtures. Carbohydr Polym. 2014;112:125-33.

(37) Tywabi Z, Deenadayalu N, Sithole B. Dissolution of South African eucalyptus sawdust wood in [Emim][OAc]/Co-solvent mixtures. J Sci Ind Res. 2017;76:166-72.

(38) Hawkins JE, Liang Y, Ries ME, Hine PJ. Time temperature superposition of the dissolution of cellulose fibres by the ionic liquid 1-ethyl-3-methylimidazolium acetate with cosolvent dimethyl sulfoxide. Carbohydr Polym Technol Appl. 2021;2:100021.

(39) Hedlund A, Theliander H, Köhnke T. Mass transport during coagulation of cellulose-ionic liquid solutions in different nonsolvents. Cellulose. 2019;26:8525-41.

(40) Le KA, Rudaz C, Budtova T. Phase diagram, solubility limit and hydrodynamic properties of cellulose in binary solvents with ionic liquid. Carbohydr Polym. 2014;105:237-43.

(41) Lei L, Lindbråthen A, Sandru M, Gutierrez MTG, Zhang X, Hillestad $M$, et al. Spinning cellulose hollow fibers using 1ethyl-3-methylimidazolium acetate-dimethylsulfoxide co-solvent. Polym (Basel). 2018;10:1-11.

(42) Müller K, Zollfrank C. Ionic liquid aided solution-precipitation method to prepare polymer blends from cellulose with polyesters or polyamide. Eur Polym J. 2020;133:109743.

(43) Bylin S, Olsson C, Westman G, Theliander H. Solvation behavior of cellulose and xylan in the MIM/EMIMAc ionic liquid solvent system: parameters for small-scale solvation. Bio Resour. 2014;9:1038-54.

(44) Adu C, Zhu C, Jolly M, Richardson RM, Eichhorn SJ. Continuous and sustainable cellulose filaments from ionic liquid dissolved paper sludge nanofibres. J Clean Prod. 2021;280:124503.
(45) Liu R, Zhang J, Sun S, Bian Y, Hu Y. Dissolution and recovery of cellulose from pine wood bits in ionic liquids and a co-solvent component mixed system. J Eng Fiber Fabr. 2019;14:1558925019838440.

(46) Luengnaruemitchai A, Anupapwisetkul C. Surface morphology and cellulose structure of Napier grass pretreated with the ionic liquid 1-ethyl-3-methylimidazolium acetate combined with either water or dimethyl sulfoxide as a co-solvent under microwave irradiation. Biomass Convers Biorefinery. 2020;10:435-46.

(47) Mao J, Abushammala H, Pereira LB, Laborie MP. Swelling and hydrolysis kinetics of Kraft pulp fibers in aqueous 1-butyl-3methylimidazolium hydrogen sulfate solutions. Carbohydr Polym. 2016;153:284-91.

(48) Mao J, Heck B, Reiter G, Laborie MP. Cellulose nanocrystals' production in near theoretical yields by 1-butyl-3-methylimidazolium hydrogen sulfate ([Bmim]HSO4) - Mediated hydrolysis. Carbohydr Polym. 2015;117:443-51.

(49) Mao J, Osorio-Madrazo A, Laborie MP. Preparation of cellulose I nanowhiskers with a mildly acidic aqueous ionic liquid: reaction efficiency and whiskers attributes. Cellulose. 2013;20:1829-40.

(50) Liu Z, Sun X, Hao M, Huang C, Xue Z, Mu T. Preparation and characterization of regenerated cellulose from ionic liquid using different methods. Carbohydr Polym. 2015;117:54-62.

(51) Shamsuri AA, Jamil SNAM. Application of quaternary ammonium compounds as compatibilizers for polymer blends and polymer composites - a concise review. Appl Sci. 2021;11:3167.

(52) Shamsuri AA, Jamil SNAM, Abdan K. Processes and properties of ionic liquid-modified nanofiller/polymer nanocomposites a succinct review. Processes. 2021;9:480. 